\title{
Les métaphores de l'eau dans Les Fleurs du Mal de Charles Baudelaire
}

\section{Mema, Laureta}

Université de Tirana, lauretamemav@gmail.com

\begin{abstract}
Resumen
Este artículo investiga el proceso de las metamorfosis del agua que primero se encaminan hacia el deseo erótico del poeta y después hacia una destinación existencial. El deseo erótico del poeta depende del agua, con el fin de dejar el mundo humano y encontrar la isla divina donde él podrá proteger su amor (« L'Invitation au Voyage »). Por otra parte, el agua va a reflejar la desesperación del poeta, destinado de amar la mujer pervertida endemoniada («Moesta et Arrabunda ») o imaginar un vampiro Venus el cual le va a asegurar solo una horca para contemplar su imagen de víctima («Un voyage à Cythère »). Además del deseo erótico, las metáforas del agua surgen cuando el sujeto medita sobre el destino humano. Ellos tratan de explicar la fatalidad de la vida desesperada de los poetas ( "L'Ennemie ») o consolarlo mientras revela su propia imagen (« L’Homme et la Mer»).
\end{abstract}

Palabras clave : agua ; Baudelaire ; viaje ; estético ; metáfora.

\section{Résumé}

Nous allons observer le processus de la métamorphose de l'eau (un élément important pour Baudelaire qui se transforme souvent en mer) pour naviguer premièrement dans le désir érotique et deuxièmement dans une destinée humaine existentielle. Le désir érotique du poète dépendra de l'eau, afin de quitter le monde terrestre et trouver l'île paradisiaque où il pourrait protéger sa vie amoureuse ("L'Invitation au Voyage »). Mais de l'autre côté, l'eau miroitera le désespoir du poète qui est destiné à aimer la femme corrompue, infernale ( "Moesta et Arrabunda ») ou à envisager la Vénus vampire qui ne lui assurera qu'un gibet pour contempler son image de victime («Un voyage à Cythère »). À part le désir érotique, on verra que les métaphores de l'eau surgissent et tourbillonnent quand le poète médite à la destinée humaine. Elles essayent d'expliquer la fatalité de la vie malheureuse du poète ( L'Ennemie ») ou ils aboutissent pour le consoler, en lui renvoyant sa propre image («L'Homme et la Mer »).

Mots-clés: eau ; Baudelaire ; voyage ; métaphorisation ; esthétique.

\begin{abstract}
This paper examines the process of water metamorphoses to firstly navigate in the poet's erotic desire and later in an existential destination. The poet's erotic desire depends on water, in order to leave the human world and find the heavenly island where he could protect his love ("L'Invitation au Voyage»). On the other hand, water will mirror the poet's despair, destined to love the corrupted infernal woman (« Moesta et Arrabunda») or envisage a vampire Venus who will assure him only a gibbet to contemplate his victimized self («Un voyage à Cythère »). Besides erotic desire, water metaphors arise when the subject meditates on human destination. They try to explain the fatality of the poet's desperate life («L'Ennemie ») or console him when revealing his proper image («L'Homme et la Mer »).
\end{abstract}

Keywords : water ; Baudelaire ; voyage ; aesthetic ; metaphor. 
Dans la critique de Théophile Gautier, Baudelaire maintient que Gautier est l'écrivain par excellence «parce qu’il est l'esclave de son devoir, parce qu'il obéit sans cesse aux nécessités de sa fonction, parce que le goût du Beau est pour lui un fatum, parce qu'il a fait de son devoir une idée fixe ${ }^{1}$. Dans cette citation Baudelaire dévoile aussi son projet esthétique. Notre poète, dévoue sa vie à un devoir unique qui ne sera que son « idée fixe ». Il décidera de créer un monde nouveau. Il est le témoin des grands architectes de la littérature, comme Hugo, Chateaubriand, Gautier, Balzac, Flaubert, Goethe, Poe, etc., qui lui ont montré la voie, le sens de la vie, l'unique possibilité de réussir une « élévation » poétique et de se débarrasser de l'ennui meurtrier, condamnation éternelle pour les terrestres. Or, pour faire vivre ce monde moderne, le poète doit mendier, se diriger vers tous ceux qui peuvent donner ce qui conviendrait aux goûts de son univers guidé par l'imagination. Le poète intériorise ce Monde imaginé qui doit «imposer tous les temps et tous les univers » ${ }^{2}$, y compris les quatre éléments : la Terre, l’eau, l’air et le soleil. Nous allons arrêter notre regard, dans la métaphorisation de l’eau, un élément important pour Baudelaire, pour naviguer premièrement dans le désir érotique du poète ( « L’invitation au voyage », « Moesta et Arrabunda », «Voyage à Cythere »), et deuxièmement dans une tentative existentialiste (« L’Ennemie », « L’Homme et la mer »).

Dans « L’Invitation au Voyage », le sujet s’adresse à une femme l'invitant à voyager vers un pays qui lui ressemble, en vue d'atteindre son propre bonheur. L'eau est vitale parce que d'abord, elle protège le monde exotique où le couple pourra goûter les plaisirs paradisiaques, même si imaginaires, mais aussi parce qu'elle contient les bateaux qui pourraient les emmener ensemble « là », désertant à jamais le monde terrestre. Le sujet lyrique invite cette femme en pleurs à rêver du rêve qui le possède, exigeant une union perfectionnée. Le pays rêvé qui ressemble à la femme a pourtant les charmes de ses yeux «traîtres », mot clé qui nous aide à comprendre pourquoi le poète voudrait s’évader dans un pays d’ordre, la trahison étant désordre. La femme donc est accusée ; elle est adorable tout en causant le désespoir du poète. Le monde qui se trouve ailleurs, entouré et protégé par l'eau («Là, tout n’est qu’ordre et beauté ») est mis en contraste avec le monde présent qui contient les yeux traîtres et souffrants. Cette union semble impossible en réalité et on verra qu’elle subsiste à être impossible même dans un rêve :

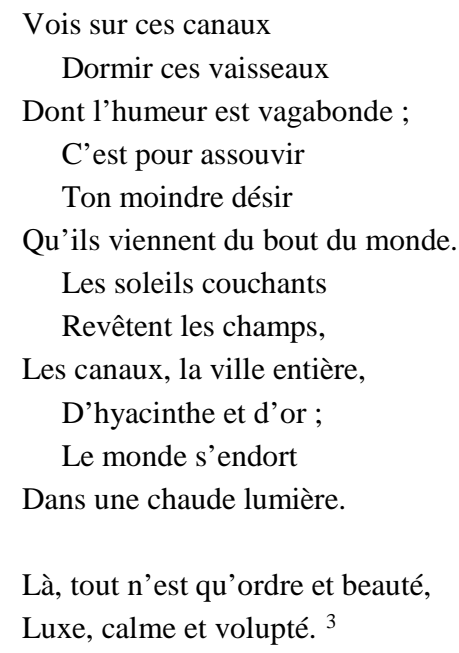

Le poète dirige son regard vers le monde du présent en ordonnant la femme de le contempler aussi. Les bateaux aux « humeurs vagabondes » dorment mais sont prêts au prochain voyage. Il lui offrira des couchers de soleil qui couvriront la ville avec leur couleur d'or. Le monde devrait s'endormir sous « une chaude lumière » pour que la belle rêve du rêve du poète, tranquillement. Donc, les soleils viennent à l'aide du poète, enveloppant le couple avec leur énergie pour qu’ils soient unis dans le rêve. Pourra-t-elle rêver le rêve du poète ou sera-t-elle toujours traîtresse ? Malgré la lumière du soleil qui englobe l'univers entiers, le désir est pareil, inassouvi. On pourrait s'attendre à ce que les couchers de soleil qui

\footnotetext{
${ }^{1}$ Baudelaire, 1980 (498).

2 Idem, « Bénédiction » (7).

${ }^{3}$ Idem, « L'Invitation au Voyage » (39).
} 
envahissent la ville, y compris les bateaux, anéantissent le désir du poète. En effet, si le monde s'endort, le poète doit être satisfait. Notons qu'elle est ordonnée à s’endormir mais lui, il reste réveillé pour ordonner l'endormante d’y aller « là ». Barbara Johnson recourt à ce poème en vue de maintenir que la poésie, contrastée avec la prose, est « unité, totalité, immortalité, et pouvoir $»{ }^{4}$. En effet, notons que les différences entre le sujet et l’objet désiré ne sont pas abolies ; c’est le sujet qui ordonne et le sujet désiré qui doit obéir en rêvant le rêve du poète qui malheureusement ne sera jamais comblé. Le langage n’assure pas le voyage puisqu'il le décrit au conditionnel ; le temps n'est pas frisé puisque on passe du présent au futur, etc. L’espace n’est pas unifiée non plus puisque le contraste entre ici et là, ou la réalité et le rêve demeure. William Franke de son côté propose que ce poème illustre un projet symboliste d’une identité complétée et réalisée par le langage. Selon lui il n’y a pas de différence entre les deux mondes comparés. ${ }^{5}$ En fait, ce monde-là existe comme possibilité d'assouvir le désir du poète. Le poète rêve d'un autre monde pour séduire la femme et indirectement s'évader dans un espace intime sans intrusion externe. Étant que les yeux de la femme sont traîtres et le pays ressemble à la femme, la trahison ne pourra pas assurer une identité parfaite. Le monde interne et externe se combinent tout en gardant l'identité d'autrui et que le titre ne décrit qu'une invitation. La poésie n’est pas vide de « substance réel » puisqu'il est clair qu’elle est guidée par le désir du poète.

L’idéologie du poète Baudelairien d’abandonner le monde vers un autre, par la médiatisation de l'eau, pour assurer l'accomplissement de la vie en couple, amoureuse et érotique à la fois, se révèle aussi « Moesta et Errabunda ». Le sujet lyrique envoi une autre invitation à la femme aimée avec le même message, fuir le présent envers un monde différent et meilleur et encore il dépendra d'elle pour le réaliser.

Dis-moi ton cœur parfois s'envole-t-il, Agathe,

Loin du noir océan de l’immonde cité

Vers un autre océan où la splendeur éclate,

Bleu, clair, profond, ainsi que la virginité?

Dis-moi, ton cœur parfois s'envole-t-il, Agathe? ${ }^{6}$

Il invite Agathe à abandonner « l’océan noir », la ville humaine, source de malheur, corruption et immoralité, pour choisir un autre océan comparé à la virginité « où la splendeur éclate ». Dans la deuxième strophe, la mer, avec sa «fonction sublime de berceuse » revêt une vision romantique qui se réfère à l'amour physique, avec un pouvoir satanique en même temps. Comme dans « L'Invitation au voyage », la mer a la fonction de berceuse et semble consoler les humains de leur destin pénible pour qu'ils puissent continuer avec leur vie damnée tout en revêtant la gardienne et la Muse du Poète qui voudrait glisser dans ses ondes vers un autre monde, exclusivement avec son aimée. Le monde présent dirigé par le remord, le crime, la douleur, est contrasté avec celui rêvé, « paradis parfumé », créé pour être aimé et n’étant que « pure volupté » :

Emporte-moi wagon! enlève-moi, frégate!

Loin! loin! ici la boue est faite de nos pleurs!

— Est-il vrai que parfois le triste cœur d'Agathe

\footnotetext{
${ }^{4}$ Johnson, Barabara : « The passage from poetry to prose seems to involve an amputation of everything which, in poetry, is erected as unity, totality, immortality, and potency. [...] Thus consisting of an extensible collection of miscellaneous properties and fragmentary descriptions, the prosaic all is metonymic rather than metaphoric, inclusive rather than exclusive, circumstantial rather than essential. The passage from essence to attribute is a passage from totality to partition; while the poetic all is as such indivisible, the prose poem's all is divided into a series of attributes whose number can be indefinitely increased without being able to exhaust the meaning of all, the sum of which the enumeration indefinitely defers (59-60)».

${ }^{5}$ William, Frank : « The external world here is totally at the service of, and has no determination or identity independently of, the innerness of desire. By thematizing the principle of identity in this way, the poem gives a lyric image of how language in fact operates in symbolist poetry - namely, by identifying itself concretely with what it represents and erasing the difference between representation and reality, the inner world and the outer. »; « On the one hand, reality puts up no more resistance : all is simply fused into unity in an exquisite and unrestricted universal harmony forged in and by language. On the other hand, the collapse of all extralinguistic reality into language leaves language empty of real substance and consequently disoriented. Without being anchored to anything real beyond itself, language has trouble maintaining even its own unity and integrity (20)».

${ }^{6}$ Baudelaire, Idem « Moesta et Arrabunda » (47).
} 
Dise: Loin des remords, des crimes, des douleurs,

Emporte-moi, wagon, enlève-moi, frégate?

Comme vous êtes loin, paradis parfumé,

Où sous un clair azur tout n'est qu'amour et joie,

Où tout ce que l'on aime est digne d'être aimé,

Où dans la volupté pure le cœur se noie!

Comme vous êtes loin, paradis parfumé! ${ }^{7}$

Donc, on rencontre frénétiquement le désir du poète de s’évader vers un autre monde où règne « la volupté pure ». Comme Barthes explique, le monde devient un ennemie, parce qu’il dérobe l'aimée à soi. ${ }^{8}$ Le fait que Baudelaire a choisi Agathe qui nous rappelle la vierge sainte, l'Agathe de Sicile, montre qu'il s’adresse à une femme qui devrait partager le désir du sujet, en vue de déserter le monde du crime et de la corruption morale. Après la lecture des dernières strophes on apprend malheureusement que le poète tombe au désespoir et que son invitation reçoit l'écho de sa mélancolie désespérée, vu qu’il est destiné à vivre avec un adversaire implacable.

La condamnation de vivre dans un monde corrompu dirigé par des femmes vampires, l'escorte de Venus Fatale, montre en effet le projet esthétique de Baudelaire de " pétrarquiser sur l’horrible » comme Sainte-Beuve l'avait bien noté, ${ }^{9}$ et comme je l'étudie aussi dans ma thèse de Doctorat, ${ }^{10}$ illustré aussi dans « Un Voyage à Cythère ». Cette fois-ci le poète sera un heureux voyageur dans le navire qui « roulait sous un ciel sans nuages, / Comme un ange enivré d'un soleil radieux ». Ce voyage, dont la description est emprunté à Nerval, ${ }^{11}$ est dessiné avec tant d'enthousiasme et une attente romantique, en imaginant même une «jeune prêtresse, amoureuse des fleurs » et qui devrait aller «le corps brulé de secrètes chaleurs, / Entre-bâillant sa robe aux brises passagères ». Le poète voit de loin son Eldorado adoré par tous les hommes, et s’imagine encore les multiples beautés qu’il devrait rencontrer. Or, son désir se renverse. On le sent dès les vers «- Cythère n’était plus qu’un terrain des plus maigres, /Un désert rocailleux troublé par des cris aigres ». Comme Nerval, le poète perçoit, « un gibet à trois branches » où il y a « un pendu déjà mur » qui se trouve dans la même situation que l'animal mourant dans « Une Charogne » :

Devant toi, pauvre diable au souvenir si cher,

J'ai senti tous les becs et toutes les mâchoires

Des corbeaux lancinants et des panthères noires

Qui jadis aimaient tant à triturer ma chair.

- Le ciel était charmant, la mer était unie ;

Pour moi tout était noir et sanglant désormais,

\footnotetext{
${ }^{7}$ Idem.

${ }^{8}$ Barthes, Roland : « The world is full of indiscreet neighbors with whom I must share the other. The world is in fact just that : an obligation to share. The world (the worldly) is my rival. I am continually disturbed by intruders : a vague connection, met by chance and who forces his way into our company, sits down at our table; neighbors in the restaurant whose vulgarity visibly fascinates the other, to the point where he is unaware if I am speaking to him or not; even an object, a book for instance, in which the other is absorbed (I am jealous of the book) Everything is irksome which briefly erases the dual relation, which alters the complicity and relaxes the intimacy : "You belong to me as well," the world says (111)».

${ }^{9}$ Sainte-Beuve : « Vous êtes, vous aussi, de ceux qui cherchent de la poésie partout; et, comme avant vous, d’autres l'avaient cherchée dans des régions toutes ouvertes et toutes différentes ; comme on vous avez laissé peu d'espace ; comme les champs terrestres et célestes étaient à peu près tous moissonnés, et que, depuis trente ans et plus, les lyriques, sous toutes les formes, sont à l'œuvre - venu si tard et le dernier, vous vous êtes dit, j'imagine : "Eh bien ! J'en trouverai encore de la poésie, et j'en trouverai là où nul ne s'était avisé de la cueillir et de l'exprimer. » Et vous avez pris l'enfer, vous vous êtes fait diable ; vous avez voulu arracher leurs secrets aux démons de la nuit. En faisant cela avec subtilité, avec raffinement, avec un talent curieux et un abandon quasi précieux d'expression, en perlant le détail, en pétrarquisant sur l'horrible, vous avez l'air de vous être joué ; vous avez pourtant souffert, vous vous êtes rongé à promener et à caresser vos ennuis, vos cauchemars, vos tortures ; vous avez dû beaucoup souffrir, mon cher enfant (312)».

${ }^{10}$ Ma thèse de doctorat, « Pétrarquisant sur l'horrible » in Les Fleurs du Mal, Eros and the art of poetry from the Troubadours to Baudelaire » poursuit justement la thèse de Sainte-Beuve. On constate qu’en réalité Baudelaire choisit sa Muse, Femme fatale, sœur de Diane, Venus d’Aubigné.

${ }^{11}$ Pichois, Claude : « La dédicace provisoire à Nerval dans ms. rappelle l’origine du poème et montre que ce dernier doit être, avec certitude, considérée comme une réponse à un texte précis de Nerval, une description de l'île de Cérigo, l'ancienne Cythère. [...]. Voici ce qu'écrivait Nerval : « Voilà mon rêve... et voici mon réveil ! Le ciel et la mer sont toujours là ; le ciel d'Orient, la mer d'Ionie se donnent chaque matin le saint baiser d'amour ; mais la terre est morte, morte sous la mains de l'homme, et les dieux se sont envolés !/ Pour rentrés dans la prose, il faut avouer que Cythère n’a conservé de toutes ses beautés que ses rocs de porphyre, aussi tristes à voir que de simples rochers de grès [...]. J'avais aperçu un petit monument, vaguement découpé ; sur l'azur du ciel, et qui, du haut d’un rocher, semblait la statue encore debout de quelque divinité protectrice... Mais en approchant d'avantage, nous avons distingué clairement l'objet qui signalait cette côte à l'attention des voyageurs. C'était un gibet à trois branches ».
} 
Hélas ! et j’avais, comme en un suaire épais,

Le cœur enseveli dans cette allégorie.

Dans ton île, ô Vénus ! je n’ai trouvé debout

Qu'un gibet symbolique où pendait mon image...

- Ah ! Seigneur ! donnez-moi la force et le courage

De contempler mon cœur et mon corps sans dégoût ! ${ }^{12}$

Si dans « Une Charogne », le poète avait dirigé son regard vers son aimée, pour lui rappeler le même destin et lui assurer traditionnellement qu'il garderait son essence et sa forme divine, dans ce poème le poète se regarde lui-même comme charogne. En ensevelissant le cœur dans une allégorie, le poète défierait-il la mort comme il le dit dans « L’Allégorie » : «Elle rit à la Mort et nargue la Débauche,[...] / Elle ignore l’Enfer comme le Purgatoire, /Et quand l'heure viendra d'entrer dans la Nuit noire, / Elle regardera la face de la Mort, / Ainsi qu'un nouveau-né, - sans haine et sans remord. » Il semble que non puisqu'il est pareil au Christ et choisit de prier Dieu. Dans le poème qui suit on rencontre Christ qui prie Dieu pareillement, mais sans espoir : « - Ah ! Jésus, souviens-toi du Jardin des Olives ! / Dans ta simplicité tu priais à genoux / Celui qui dans son ciel riait au bruit des clous /Que d'ignobles bourreaux plantaient dans tes chairs vives, ». ${ }^{13}$ On remarque la similarité entre notre poète et Dieu. Comme Christ, le poète garde romantiquement l'humanité dans son cerveau. À la compagnie de la Venus, que Jean Starobinski souligne avec raison mélancolique, ${ }^{14}$ et donc à la lignée du projet esthétique romantique, le poète risque de métamorphoser la douleur dans une beauté esthétique puisque la mélancolie anéantit le travail étant synonyme d'état de mort. Ainsi faut-il comprendre la prière du poète dirigée vers Dieu, pour l’aider à n’être pas le guignon qui perdrait le diadème divin prétendu dans la « Bénédiction ».

En effet, Baudelaire a assemblé l'histoire de Nerval et la réflexion de De Quincey dans « Un mangeur d'Opium »: « Vous savez maintenant que l'apparition n'est que votre propre reflet et qu'en adressant au fantôme l'expression de vos secrets sentiments, vous en faites le miroir symbolique où se réfléchît à la clarté du jour ce qui autrement serait resté caché à jamais ». Notons que la prière n'est pas de sauver le poète mais de « contempler », ce qui veut dire travailler, même s'il n'est qu'un mort joyeux : « Je hais les testaments et je hais les tombeaux ; / Plutôt que d'implorer une larme du monde, /Vivant, j'aimerais mieux inviter les corbeaux/ À saigner tous les bouts de ma carcasse immonde. ». John Jackson souligne que la proximité du sujet à l'expérience de la mort permet au poète de prendre une prise de conscience de soi, «la nouveauté que Baudelaire introduit dans la tradition poétique du discours allégorique de la mort ou sur la mort, c’est que celle-ci ne surgît le plus souvent dans ses poèmes qu'en rapport explicite avec le je qui l'interpelle ou qui en est interpellé ». ${ }^{15}$ Ainsi, Jackson reprend la thèse de Marcel Proust (dont Yves Bonnefoy doit être certainement inspiré quand il dit que Baudelaire voit le cadavre non seulement de l'extérieur mais aussi de l'intérieur) : «À coté d'un livre comme Les Fleurs du Mal, comme l'œuvre immense d’Hugo paraît molle, vague, sans accent ! Hugo n’a cessé de parler de la mort, mais avec le détachement d'un grand mangeur et d'un grand jouisseur. Peut être hélas ! Faut-il contenir la mort prochaine en soi, être menacé d'aphasie comme Baudelaire, pour avoir cette lucidité dans la souffrance véritable. » ${ }^{16}$

À travers l'œuvre on voit le désir obsédé du poète qui rencontre «La Béatrice » fatale. Il rêve d'une île innocente, un paradis qui propose des plaisirs enfantines dans un panorama pastorale: «Les courses, les chansons, les baisers, les bouquets, / Les violons vibrant derrière les collines, /Avec les brocs de vin, le soir, dans les bosquets ». ${ }^{17}$ Ce monde rêvé devient torturant puisqu'il ne peut se trouver que dans le désir, les « cris plaintifs » d'un homme assoiffé de bonheur mais ne rencontrant que des bêtes ivres de plaisirs charnels. Ce n'est que dans le corps de la femme, sa beauté idyllique, qu'elle devient réalisation de ce voyage tant rêvé :

\footnotetext{
12 Baudelaire, Idem, « Voyage à Cythère » (87).

${ }^{13}$ « Le reniement de Saint Pierre ».

${ }^{14}$ Jean Starobinski compare Baudelaire avec Schiller disant que « Dans l'élégie selon Schiller, la nature et l'idéal sont objets de deuil, car la nature est représentée comme perdue et l'idéal comme non encore atteint. Spleen et Idéal, le sous-titre de la première partie des Fleurs, correspond d'assez près aux catégories schillériennes (57) ».

${ }^{15}$ Jackson, John (22).

${ }^{16}$ Proust, À propos de Baudelaire (9).

${ }^{17}$ Idem, « L'invitation au Voyage » (39).
} 
Que j'aime voir, chère indolente,

De ton corps si beau,

Comme une étoffe vacillante,

Miroiter la peau !

Sur ta chevelure profonde

Aux âcres parfums,

Mer odorante et vagabonde

Aux flots bleus et bruns,

Comme un navire qui s'éveille

Au vent du matin,

Mon âme rêveuse appareille

Pour un ciel lointain. ${ }^{18}$

Dans « Le Beau navire », la femme ressemble avec son costume à un navire ( «Quel poëte oserait, dans la peinture du plaisir causé par l'apparition d’une beauté, séparer la femme de son costume? » ${ }^{19}$ ): «Quand tu vas balayant l'air de ta jupe large, / Tu fais l'effet d'un beau vaisseau qui prend le large, / Chargé de toile, et va roulant / Suivant un rythme doux, et paresseux, et lent. ${ }^{20}$ Ce navire est porte-parole du rêve du poète qui part vers l'autre monde même si vivant dans la foule de la modernité, « l’océan noir » : « Tes nobles jambes, sous les volants qu'elles chassent, / Tourmentent les désirs obscurs et les agacent, / Comme deux sorcières qui font /Tourner un philtre noir dans un vase profond. » À part les métaphores érotiques, Baudelaire revient à la métaphorisation de l'eau quand il médite à la destinée humaine. Dans «L’Ennemi », le poète se pose la même question pénible sur la réussite de « son devoir » : « Et qui sait si les fleurs nouvelles que je rêve / Trouveront dans ce sol lavé comme une grève / Le mystique aliment qui ferait leur vigueur ? ». Les terres sont « inondées » par « le tonnerre et la pluie » qui « ont fait un tel ravage » et « l'eau creuse de trous grands comme des tombeaux ». « Le Temps mange la vie ! », mais pas seulement la vie du poète, la vie de l'œuvre aussi ; cette œuvre qui n’est même pas commencée et dont Baudelaire rêve obsessionnellement. Dans la correspondance, Baudelaire répète à sa mère que le temps marche trop vite et qu'il a peur de ne pas finir l'œuvre dont il est capable. En fait, Baudelaire avait des ambitions comparables à celles de Hugo. Les titres l'illustrent bien. Les Fleurs du Mal, ne sont rien comparées aux autres fleurs qu'il doit planter pour que le lecteur puisse voir finalement la Pyramide que « lui seul » peut construire. Cependant, comme Claude Pichois l’a remarqué, le temps est un prétexte, qui cache une raison capitale qui angoisse le poète : c'est l'angoisse d'une inspiration maigre. ${ }^{21}$ Donc, si la marche funèbre du temps qui « mange la vie » suggère une prise de conscience de soi comme une finitude prochaine, toujours mise en question et contestée, cette prise de conscience est fortement liée aussi avec la croissance des « fleurs nouvelles » dont le poète « rêve ». Or, même si le poète sera mort, comme dans « le Flacon », il exige une postérité, dont il croit fortement, même si la critique a voulu s’en débarrasser. ${ }^{22}$

Dans «L'homme et la mer» l'auteur perçoit l’analogie de la nature humaine avec celui de la mer. En effet le poète se trouve mal à l'aise dans la cité humaine où il ne vit que comme un Albatros captivé dans un navire. On constate que la métaphore de l'eau n'est pas trop loin de celle du sang trouvé dans le " pauvre Diable, habitant de Cythère », et dans son propre cœur d’un mort éternel puisqu’elles sont liées avec les mêmes connotations de souffrance («plainte indomptable

\footnotetext{
${ }^{18}$ Idem, « Le Serpent qui danse », (22).

${ }^{19}$ Idem, « Le Peintre de la vie moderne : X -La Femme », (809).

${ }^{20}$ Idem, « Le Beau Navire ».

${ }^{21}$ Pichois, Claude : « La présence de Hugo gêne Baudelaire qui, obligé de confesser la grandeur et la vitalité de son ainé, tourne facilement celles-ci en dérision. L’empire de l'un semble menacer le petit canton de l'autre ; mais si ce petit canton était riche de tous les ors, s'il détenait dans ses coffres les bijoux perdus de l'antique Palmyre ? Baudelaire, là-dessus n’a jamais pu se rassurer. (...) Le vertige de la page blanche, la répulsion devant la page blanche. De cette hantise, de cette phobie, ressentie par Baudelaire même pendant ses années les plus favorables, on pourrait longuement examiner les causes diverses : le tempérament, le caractère, les tristes habitudes de procrastination prises pendant la jeunesse, les progrès sournois de la syphilis, les ravages dus à l'alcool et au laudanum, l'impression, enfin, que tout est dit et que naitre après Lamartine, après Hugo, après Musset, après Gautier, c'est venir trop tard, c'est être désigné pour l'exploitation poétique de l'enfer et la culture en serre des fleurs maladives, tâche bien pénible : « Pour soulever un poids si lourd, / Sisyphe, il faudrait ton courage ! ».

${ }^{22}$ Baudelaire, Idem : C’est cet admirable, cet immortel instinct du Beau qui nous fait considérer la Terre et ses spectacles comme un aperçu, comme une correspondance du Ciel. La soif insatiable de Tout ce qui est au-delà, et que révèle la vie, est la preuve la plus vivante de notre immortalité, c’est à la fois par la poésie et à travers la poésie, par et à travers la musique, que l'âme entrevoit les splendeurs situées derrière le tombeau ; et quand un poème exquis amène les larmes au bord des yeux, ces larmes ne sont pas la preuve d'un excès de jouissance ; elles sont bien plutôt le témoignage d'une mélancolie irritée, d'une postulation des nerfs, d'une nature exilée dans l'imparfait et qui voudrait s'emparer immédiatement, sur cette terre même, d’un paradis révélé (498)».
} 
et sauvage »), richesse, liberté ou Mort. Cependant, la litote « Et ton esprit n'est pas un gouffre moins amer », suggère que l’homme se console en contemplant son image, le contraire de ce qui s'est passé à Cythère.

\footnotetext{
Vous êtes tous les deux ténébreux et discrets: Homme, nul n’a sondé le fond de tes abîmes; Ô mer, nul ne connaît tes richesses intimes, Tant vous êtes jaloux de garder vos secrets!

Et cependant voilà des siècles innombrables Que vous vous combattez sans pitié ni remords, Tellement vous aimez le carnage et la mort, Ô lutteurs éternels, ô frères implacables!
}

L'homme et la mer se caractérisent par l'infini, les ténèbres, les abîmes, les secrets, les richesses. Ils ont le même goût pour les batailles, le carnage et la mort. Baudelaire réclame le goût du macabre, que Jackson interprète comme une impossibilité de dépasser le désespoir. ${ }^{23}$ Or, cette retrouvaille de l’espoir dans la Beauté, ou dans la Mort, qui est encore une représentation de la Beauté, nous semble la preuve que Baudelaire a connu un «tel dépassement ». Baudelaire souligne que le désespoir pour un poète c’est le suicide : « La lutte et le révolte impliquent toujours une certaine quantité d'espérance, tandis que le désespoir est muet ». ${ }^{24}$ Mais qu'est-ce que c'est ce dépassement du poète: « Plonger au fond du gouffre, Enfer ou Ciel, qu'importe ? / Au fond de l'Inconnu pour trouver du nouveau ! ».

Lisant sa correspondance, on comprend vite que le Baudelaire se sent accablé par les critiques et le travail qu’il n’arrive pas finir. Or, ce qui nous surprend le plus c'est cette incroyable force, presque despotique, cette incroyable confiance dans la littérature, dans le Beau, cet amour unique pour l'art. Baudelaire sait qu’il torture sa mère et soi-même, il sait qu'il a choisi une existence misérable de littérateur non apprécié ni par le public, ni par la critique, ni par ses maîtres idoles, mais il insiste faire « son devoir ». C’est exactement en ceci que réside la compassion de Baudelaire pour l’Humanité. Il n’a pas brûlé son œuvre, il n’est pas parti dans une île pour vivre tranquille à l'exil, protégé par la mer tranquille, il est demeuré volontairement dans le monde de l’Enfer, pour en tirer de la boue l’or qui réjouira pour toujours les esprits qui recherchent le Beau, le « Paradis révélée sur terre » même s’il vient d’un océan noir qu’il abhorre ( « Je te hais, Océan ! tes bonds et tes tumultes, / Mon esprit les retrouve en lui ; ce rire amer / De l'homme vaincu, plein de sanglots et d’insultes, / Je l'entends dans le rire énorme de la mer »). Dans le quatrième « projet de préface pour Les Fleurs du Mal » le poète aspire à un sommeil tranquille. ${ }^{24}$ Peut-être, croyait-il qu'il pourrait se réveiller quand un esprit « comme lui », " amoureux du Beau » savait « entrer dans les gouffres » pour « apprendre à l'aimer », mais « sans se laisser charmer »?

Comme tu me plairais, ô nuit ! sans ces étoiles

Dont la lumière parle un langage connu !

Car je cherche le vide, et le noir, et le nu !

Mais les ténèbres sont elles-mêmes des toiles

Où vivent, jaillissant de mon œil par milliers,

Des êtres disparus aux regards familiers.

\footnotetext{
${ }^{23}$ Jackson : « La conscience du désespéré est susceptible d’un degré de lucidité supplémentaire qui transforme alors le désespoir en un espoir nouveau, le libre arbitre venant rédimer ce péché par l'acte de sa volonté. Baudelaire, lui, ne connaît pas la possibilité d’un tel dépassement. S’il partage profondément le sentiment exprimé dans la logique de cette désespérance, il le subit aussi à la manière d’une fatalité qui l'angoisse et devant laquelle il semble parfois irrémédiablement désemparé - comme s'il ne parvenait pas alors, pour reprendre les termes du philosophe danois, à dépasser le stade esthétique d’une conscience de soi ne trouvant sa résolution qu'au stade du religieux. Fasciné par son « culte des images », Baudelaire semble aussi bien se trouver à sa merci (78)».

${ }^{24}$ Baudelaire, (292) : « J'aspire à un repos absolu et à une nuit continue. Chantres des voluptés folles du vin et de l'opium, je n’ai soif que d’une liqueur inconnue sur la terre, et que la pharmaceutique céleste elle-même ne pourrait pas m’offrir ; d'une liqueur qui ne contiendrait ni la vitalité, ni la mort, ni l'excitation, ni le néant. Ne rien savoir, ne rien enseigner, ne rien vouloir, ne rien sentir, dormir et encore dormir, tel est aujourd’hui mon unique vœu. Vœu infâme et dégoutant, mais sincère ».
} 


\section{Références bibliographiques}

BARTHES, Roland. (1978). A lover's discourse: fragments. New York : Hill and Wang,.

BAudelaire, Charles (1975-53). Euvres Complètes, Ed. Claude Pichois ; Jean Ziegler. Paris: Gallimard.

Bonnefoy, Yves (2000). Baudelaire: la Tentation de l'Oubli. Paris : Bibliothèque nationale de France.

FrANKE, William (2001). « The linguistic turning of the symbol: Baudelaire and his French symbolist heirs », dans Baudelaire and the Poetics of Modernity. Ed. Patricia A Ward; James S Patty. Nashville : Vanderbilt University Press,.

GAUTIER, Théophile (2000). L'œuvre complète. Paris : H. Champion.

Hugo, Victor ; Pierre Albouy. Euvres Poétiques. Paris : Gallimard, 1964-1978.

JACSON, John E (1982). La Mort Baudelaire: Essai sur Les Fleurs du Mal. Neuchâtel : À la Baconnière.

JoHnson, Barbara (1985). «Poetry and Its Double: Two Invitations au voyage » dans The Critical Difference: Essays in the Contemporary Rethoric of Reading. Baltimore : Johns Hopkins UP, pp. 23-51.

LABARTHE, Patrick (1999). Baudelaire et la tradition de l'allégorie. Genève : Droz.

Pichois, Claude (2005). L’Atelier de Baudelaire : «Les Fleurs du Mal ». Paris : Honoré Champion.

Pound, Ezra (1954). The Literary Essays of Ezra Pound. London : Faber.

Proust, Marcel (2006). À propos de Baudelaire. Lettre à Jacques Rivière. Paris : Sandre.

StARovinski, Jean (1989). La Mélancolie au Miroir : Trois Lectures de Baudelaire. Paris : Julliard. 